Accepted (peer-reviewed) version of article It is authorized for self-archiving in a Scholarly Collaboration Network (SCN), including ArXiv, upon publication. Formatted by the author to enhance readability. 
Published in Journal of Cognition and Culture 21 (2021) 449-468.

\title{
Numerical Origins: The Critical Questions
}

\author{
Karenleigh A. Overmann \\ University of Colorado, Colorado Springs
}

\begin{abstract}
My propositions are elucidatory in this way: he who understands me finally recognizes them as senseless, when he has climbed out through them, on them, over them. (He must so to speak throw away the ladder, after he has climbed up on it.) (Wittgenstein, 1933, Proposition 6.54)
\end{abstract}

Trying to understand the origin of numbers through symbolic forms realized and refined over thousands of years, or by means of the innate, inchoate, inarticulate sense of quantity we share with other species, lands us in much the same position as the philosopher using Wittgenstein's metaphoric ladder. In the first case, we have climbed up very far, the ladder has long since been kicked away, and we are left wondering just how we achieved the rare and elevated heights in which we find ourselves. In the second, there is no ladder, and we marvel at a seemingly unbridgeable gap.

To recognize that a ladder is needed and then put one back where one belongs, we review four perspectives on numeracy, the ability to reason with numbers. As argued, if we are to achieve an answer on numerical origins, the nativist, linguistic, embodied, and extended perspectives must address one or more critical questions, and this will involve working across the boundaries of the different disciplines investigating numeracy.

\section{The Nativist Model}

Nativists see the innate sense of quantity known as numerosity as both necessary and sufficient for numeracy. Necessity is reasonable, given that numerosity is demonstrated by all humans, exhibits the same functionality across and despite significant differences of culture and numerical elaboration, and has a pervasive and continuing influence on numeracy (Gordon, 2004; Henrich et al., 2010; Malone et al., 2019; Pica et al., 2004). These circumstances suggest that numerosity is both foundational to, and a key determinant of, numeracy as it develops in humans (Nieder, 2017a,b). The converse - the claim that numerosity isn't necessary for numeracy - may be unfalsifiable, since numerosity has been demonstrated by all the vertebrate and invertebrate species tested for it to date.

Sufficiency is a different matter. If numerosity were sufficient for numeracy, then all human societies should have numbers because all humans have numerosity. Yet not all human societies have numbers: The Amazonian Pirahã are said to lack them (Everett, 2005; Frank et al., 2008), though this anumerical absoluteness has also been contested on the grounds that they may have terms for one and two (Everett, 2013; Nevins et al., 2009). Sufficiency also doesn't explain why some societies develop highly elaborated numbers and others relatively few. Neither does sufficiency explain the variability observed within particular cultural traditions over time. In the Western tradition over the past thousand years, one, two, and zero became numbers, Hindu-Arabic numerals replaced Roman ones, and calculating with algorithms involving mental judgments supplanted the mechanical exchange of values on counting boards (Evans, 1977; Klein, 1992; Pullan, 1968; Reynolds, 1993; Rotman, 1987). This variability suggests an elaborational 
mechanism other than whatever aspects of numeracy are innate to numerosity, arguing against sufficiency. Simply, the Wittgensteinian ladder connecting numerosity to numeracy is missing.

The question of what else might need to be added to numerosity to yield numeracy in humans is debated, not just in terms of what that something else might be (i.e., what kind of a ladder?) but whether it is necessary in the first place (is a ladder really needed?). In arguing both need and kind, the biologically endowed ability to appreciate non-symbolic quantity has been described as being distinct from, though the basis for, its culturally mediated elaboration as exact symbolic quantification and arithmetic in humans (Núñez, 2017a,b). In arguing against both need and kind, the nativists have unequivocally rejected the need for cultural mediation, claiming the biological endowment is sufficient in and of itself (e.g., Nieder, 2017a,b). The rejection effectively equates not just numeracy and numerosity but the resultant concepts themselves. Here the need for laddering is obviated by denying the end conditions differ.

For example, honeybees were recently argued to possess not just the ability to differentiate absence from presence but "the conceptual number of zero" as "an empty set quantitatively [falling or positioned] along the numerical continuum” (Howard et al., 2018, pp. 1-2). The bees' behavior was interpreted as demonstrating an "understanding that zero is lower than one," with their performance deemed "at a level consistent with that of nonhuman primates" and parallel to that of "preschool children” (Howard et al., 2018, pp. 1-2). The last claim is particularly strong, given that the preschool children in question are WEIRD (Western, Educated, Industrialized, Rich, and Democratic; Henrich et al., 2010) and exhibit not just concepts and skills thought to be innate but those acquired from several years of linguistic and cultural exposure to a highly elaborated number system (Barth et al., 2005; Gilmore et al., 2010).

The biological emergence of a conceptual understanding of zero has been claimed to involve four stages, characterized as synthesized from historical, developmental, neurophysiological, and comparative data and purported to be broadly descriptive of, and summarily applicable to, these domains, despite their disparate natures (Nieder, 2016). As described, the first stage involves the recognition of an absence, the second responds to it without giving it numerical meaning, the third assigns it a "quantitative meaning ... represented as an empty set at the low end of a numerical continuum or number line," and the fourth represents the concept symbolically (p. 831). Stage 3 corresponds to bees' conceptualization of zero, as inferred from observations of their behavior and as interpreted in terms of how humans understand zero (Howard et al., 2018), Stage 4 to the way human six-year-olds acquire the concept, as assessed by their ability to discuss and apply it with knowledgeable adults (Nieder, 2016).

For humans, the stages are arguably more applicable to the ontogenetic acquisition of Western number concepts than reflective of their historical realization. Zero is a WEIRD number, and before it was WEIRD, it was NERD (Non-western, Elaborate, Rich, and Despotic), emerging from the mathematical tradition of Babylon, whose own numerical antecedents lie deep in Neolithic Mesopotamia and the Paleolithic Levant. Four thousand years ago, Babylonian mathematicians inserted blank spaces to align the values of columnar numbers; in India 2500 years later, these spaces became a metasign marking the absence of any number, and over the last 1000 years in the West, the metasign acquired new meaning as a sign for a number with a specified value, a specific place in the ordinal sequence, and unique characteristics like an inability to act as a divisor (Joseph, 2011; Rotman, 1987). The last two historical stages seemingly conflate or invert Stages 3 and 4, as biologically described, since symbolic representation preceded the realization of a quantitative meaning. 
Equating concepts between bees and humans implies two things: First, bees must intuitively and spontaneously grasp a concept from interacting with the natural environment that humans realized only after manipulating numerical information in the specific form of handwritten notations over thousands of years, and before that, unwritten material forms over tens of thousands of years. Second, bees must realize the concept individually, but-given that human societies have invented zero only a handful of times-individual humans do not. The questions posed by these implications are these: If a Stage 3 concept of zero is innate to species as phylogenetically remote from us as bees, why does it take so much time and effort for us to develop a comparable version? Are the things that differentiate us from other species - particularly language and culture-merely epiphenomenal in this regard, or do they actually slow us down?

If human children acquire a preexisting cultural concept like zero as a function of ontogenetic maturation and pass through the stages of understanding it on that basis, surely this cannot be the correct explanation for zero's socio-historical emergence. For one thing, it would resurrect Piaget's (1928) belief that ontogenetic differences in conceptualization can be applied to the level of societies, an idea that has rightly fallen out of fashion. Notably, the socio-historical process was one in which a cultural form-writing-was central to the (re)conceptualization process, suggesting cultural mediation is appropriately identified as the elaborational mechanism. Similarly, as defined, Stages 3 and 4 imply a role for cultural mediation: Quantitative meaning, symbolic representation, and a numerical continuum entail cultural ideas about numbers, symbols for numbers, and a linear organization to the way numbers are envisioned. These things are not characteristic of emerging number systems, which lack both a concept of zero and symbols for numbers, and may not have a mental number line (Núñez, 2011). In other words, some sort of laddering is needed - if not for bees, then for humans, for whom numerosity alone doesn't appear sufficient for realizing the later stages.

For the nativist view, the critical question may not be how much of numeracy is innate and shared between humans and other species, but rather, whether, how, and why human and nonhuman numeracy differ. If we accept that the human symbolic conceptualization of zero differs from how, what, and why bees might think about naturally occurring absence, the qualitative and quantitative differences between human communication and material culture and their counterparts in non-human species suggest investigating linguistic and cultural mediation as potential factors.

\section{The Linguistic Model}

Linguists claim language as necessary and sufficient for numeracy. If language were necessary for number concepts, then as humans are the only species with language, by definition, only humans would have concepts of numbers - a conclusion not only diametrically opposed to what the nativists claim, but which also brings into question the relevance of the comparative data. The converse is true as well, since the nativist view effectively precludes a role for language. However, since all human societies have language but not all have numbers, language doesn't appear sufficient. Moreover, since all human societies have both language and numerosity, the anumeracy of some societies means that language and numerosity are not conjointly sufficient either.

The linguistic model takes several forms. One, perhaps outdated, considers numbers a subset of the language faculty: The "human number faculty [is] essentially an 'abstraction' from human language, preserving the mechanism of discrete infinity and eliminating the other special 
features of language” (Chomsky, 1988, p. 169), with discrete infinity being the ability to generate near-infinite novel combinations from finite sounds and meanings. Another variant sees both language and numbers as informed by an underlying computational capacity responsible for properties like generativity, with other features-possibly including numerosity, though this isn't specified-added subsequently (Chomsky, 2004). Numerosity has no role in the first variant, which ignores the complex quantical behaviors of alinguistic species and their implication that numeracy has a basis other than language. Numerosity isn't necessarily excluded from the second; however, since the computational basis shared by language and numeracy is presumably not numerosity, it shares the same flaw. Both struggle to explain societal anumeracy, conjecturing a binary mechanism that either switches on numbers or fails to do so (Chomsky, 2004; Hurford, 1987). This in turn assumes numbers are an all-or-nothing proposition, a monolithic construct that glosses over non-trivial differences in conceptual content, structure, and organization across cultural number systems, as well as change within particular traditions over time.

A third variant identifies recursion as the sine qua non for both language and numbers (Fitch et al., 2005; Parker, 2006). Recursion, claimed to be the "only uniquely human component of the faculty of language" (Hauser et al., 2002, p. 1569), is the ability to embed words, clauses, or phrases beside or within similar structures. In mathematics, recursion is often considered the mechanism whereby the successor function $(n+1)$ generates new numbers (Odifreddi, 1992; Reuland, 2010), though arguably, succession is an iteration, as it lacks key qualities of recursion: self-reference, the ability to increase embedding depth, and a terminating condition (Karlsson, 2010; Taraban \& Bandara, 2017). In language, recursion has been credited as the mechanism of discrete infinity; in spoken numbers, names for higher numbers like twenty-two are recursively concatenated from "a finite lexicon of atomic units or words" like two and ten (Ott, 2009, p. 256). The Pirahã language is often highlighted in discussions of recursion and numeracy because it has been described as lacking both, with the lack of recursion claimed to explain Pirahã anumeracy (Everett, 2005; Frank et al., 2008). These claims have also been contested, recursion because it is used in Pirahã storytelling and demonstrated in speaking Portuguese, numbers because Pirahã may include terms for small numbers (Everett, 2013; Nevins et al., 2009; Sakel, 2012).

Granted, the ability to generate number words through recursive concatenation is an important linguistic contribution to numeracy. It's still not clear that an absence of recursion would prevent the subitizable quantities from being named, as they are immediately available to human perceptual experience, and indeed, Pirahã may have terms for one and two. Certainly, initial terms for numbers are often descriptive of material forms instantiating appreciable quantities, suggesting that while generativity is important, so too is the descriptive use of language, and so are the material forms that instantiate quantities and the perceptual system that appreciates them. Similarly, if recursion were the mechanism generating names for the initial numbers-say, one through ten-naming would presumably continue beyond the subitizable range, rather than invariably stopping at its upper limit. Yet the first words for numbers across languages and cultures are one, two, (occasionally) three or about three or four, (infrequently) four, and many. These terms are consistent with our perceptual experience, which recognizes the subitizable quantities one to about three or four and above that appreciates quantity differentials if they fall above a threshold of noticeability. Presumably, naming stops because we name the quantities we can see, and we can't and don't name quantities we cannot see. Recursion doesn't appear to influence this, but then, the comparative evidence suggests that numerosity has the same functionality regardless of whether or not language itself is present. 
Yet another variant claims “Without using language, we still can't go ["higher than the subitizing range of about four"]” (Hurford, 2007, pp. 91-92). A role for numerosity is implied in differentiating subitizable and non-subitizable quantities; the distinction occurs in a discussion of numerosity and conceptualization, identified as abilities possessed alike by humans and nonhuman species. The discussion reasonably proposes that non-human species may be limited conceptually to subitizable quantities. It less plausibly positions the conceptual limit as a matter of not having language, which seemingly assumes either that language is the mechanism whereby concepts of exact non-subitizable quantities are realized or that concepts don't exist unless and until they assume linguistic form (Wittgenstein, 1933). Granted, when number concepts exist in a culture, language provides an acquisitional mechanism (Cantlon et al., 2006; Dehaene, 2007); nonetheless, this doesn't answer the question of how such concepts emerge in the first place, nor does it entail the mechanism is wholly linguistic. In the absence of preexisting concepts for any non-subitizable quantities, we must still ask how and why we would name quantities that we lack the perceptual capacity to discriminate.

To understand how and why we might transcend our perceptual limits, it's helpful to recognize both numerosity and numeracy as strongly visual, with visual perception informing numerical conceptualization, comprehension, learning, visualization, performance, and epistemology (Crollen et al., 2011; Dreyfus, 1991; Giaquinto, 2007; Kaput, 1987; Nelsen, 1993; Tang et al., 2006). Numerosity and numeracy are also strongly alinguistic, as numerosity is not influenced by whether or not language for numbers is available (Henrich et al., 2010), and numerical thinking involving regions of the brain other than those implicated in language (Amalric \& Dehaene, 2016; Brannon, 2005; Varley et al., 2005). The visual, alinguistic nature of numerosity and numeracy suggests that numerical conceptualization is rooted in what we perceive visually and can manipulate into new, visually appreciable patterns, occasioning the need for linguistic labeling. Instructive in this regard are the first non-subitizable terms that typically emerge across languages and cultures: They are often five and ten, and the words for them tend to be closely associated with the fingers, hands, or fists. The hand is likely used as a material exemplar because it is visually salient, neurally interconnected with numerosity (Overmann, 2021a; Roux et al., 2003), and able to bridge the internal and external domains of experience (Gallagher, 2013; Malafouris, 2013). Simply, we name the quantities we can see and then use a material form - a ladder, in our metaphoric motif; the hand, in actuality — to attain ones outside our perceptual limits.

When numbers first emerge, they depend less on language and rely more on material structures than is true of conceptual domains like color. Just like we appreciate quantity, we perceive color. Language lets us point to and designate quantities and colors alike, and refer to them in their absence. But with numbers, we can also represent quantity with our bodies or with objects in the immediate environment. Displaying the fingers and making marks on the ground have no counterparts when it comes to representing color. And as numbers elaborate, it becomes increasingly useful to get language out of their way. As written signs, numbers are more usable without a phonetic component (the difference between 7 and seven), with operational signs $(+, \sqrt{ }$ ) ultimately achieving similar semantically meaningful, non-phonetically specified, concise visual forms (Chrisomalis, 2010; Schulte, 2015). Some question the role of language in mathematics altogether, preferring equations, diagrams, and pictures because of their visually appreciable forms (Landy, 2010; Silver, 2017).

For the linguistic view, the critical question may be how and why functions like generativity interact with language-external influences on, and mechanisms of, conceptual content 
and structure. It's difficult to imagine concepts of non-subitizable quantities as emerging from words alone; while naming is important, so too is there being something to name, and both the material form and the ability to perceive and rearrange its quantity precede the emergence of verbal labels-phylogenetically, historically, and ontogenetically. The centrality of material forms to the conceptualization process implies that they are potential sources of concepts and mechanisms of conceptual change.

\section{The Embodied Model}

In the embodied model, numbers are abstract ideas conceived through conceptual metaphor, a cognitive mechanism that "allow[s] us to reason about one kind of thing as if it were another"; this is the human capacity for symbolic reference, the ability "to associate physical symbols (or words) with numbers (which are conceptual entities)” (Lakoff \& Núñez, 2000, pp. 6, 52; also see Núñez, 2017a). Conceptual metaphor builds upon the idea of conceptual blending, which proposes that (internal/mental) representational structures interact to produce novel structure (Fauconnier, 1997; Fauconnier \& Turner, 2002). The model of conceptual blending consists of four interacting mental spaces: two input spaces, differentiated as source and target; an optional generic space that captures higher-level structure shared by the two inputs; and a blended space wherein the inputs interact. Blends inherit some of their structure from their inputs, whose interaction can also yield novel structure. To generate numerical concepts, conceptual metaphors blend the ability to appreciate non-symbolic quantity with the visual experience of quantity in collections of objects, with the results expressed linguistically (Lakoff \& Núñez, 2000, pp. 54-68).

Under the embodied model, physical experience and conceptual metaphorizing are added to numerosity and language, as in the nativist and linguistic models. Experiencing collections of objects is the basis for forming concepts of their quantity, adding to and taking away from them the basis for concepts of addition and subtraction, and so on. However, these conditions still do not explain why some societies have few or no numbers, since all humans have numerosity and language, experience physical objects, and produce conceptual metaphors. Attesting to the latter are color terms in Pirahã: "Blood” is the word for red; "immature," green/blue; "see through it," white; "shaded” or "out of the sun," dark (Everett, 2007, p. 21). As they are based on the physical experience of objects, they fit the definition of conceptual metaphor, leaving Pirahã anumeracy unexplained.

Given their plausibility and empirical support, it's difficult to argue against the ideas that embodiment influences cognition, blending and metaphorizing are reasonable functional models of conceptualization and linguistic expression, collections of objects inform concepts of cardinality and addition, Western numbers are culturally and symbolically mediated, and symbolling is important. Accordingly, the model's assumptions and omissions are the focus here: By admitting a role for the body and hinting at one for external material forms, the embodied model implicitly lets cognition start to "leak" outside the head. However, it maintains a tight, neurocentric focus on the brain: Symbolling is envisioned as a brain-bound process, with material forms assumed to have little role in the process or its outcomes. Symbolling is also assumed to be central to numerical conceptualization and elaboration. Granting this is true of WEIRD numbers, it's not true of emerging numbers, which are represented non-symbolically, or at least less symbolically, depending on where symbolling is construed to start. The model largely omits the emerging number systems of small-scale, traditional societies, despite their potential relevance to an argument about how numbers emerge; the authors cited only a single ethnomathematical reference, 
an anthology focused on challenging Eurocentrism in mathematics education. Finally, the model omits the many material devices used to represent and manipulate numbers across emerging and elaborated number systems alike. The result is heavily skewed toward highly elaborated, symbolically expressed numbers, with only a part of a ladder-collections of objects— to explain how these were achieved.

Though counting devices are not mentioned, representing quantity with the fingers or a set of marks could be considered a form of symbolling (i.e., in using one thing to represent another). This behavior is unique to humans, for even our closest primate relatives don't count on their fingers, despite sharing our pentadactyl limbs and much of our neuroanatomy. Using the hands to instantiate and express quantity highlights the importance, if not of language per se, then of social abilities to communicate and use material culture. Nonetheless, it seems a thin basis upon which to designate the resultant representations as symbolic. A distinction must still be drawn between symbolic and non-symbolic representational forms, with some thought given to the way the former emerges from the latter. For example, the word five is symbolic in a way the phrase all the fingers on one hand is not. A symbol, after all, is a conventional representation whose form is unconnected to its meaning. If five and all the words in the phrase are themselves arbitrary associations of sound and meaning, the phrase's referent fingers and the quantity they instantiate are not. In describing and drawing attention to the hand, the phrase all the fingers on one hand is iconic and indexical, iconic because it depicts its referent, indexical because it points to it.

The hand can be directly involved in representing numbers non-symbolically: The fingers might be used with spoken numbers, either for clarity or emphasis, or the fingers used alone. Fingers might also be accompanied by a verbal phrase that merely mentions the hand as it represents a number, either because the speaker doesn't know or have names for numbers, prefers to avoid their long, cumbersome names, or chooses not to name them when communicating across languages. The non-verbal communication of quantity through the hands is subserved by the fact that the fingers do more than represent iconically: Five fingers instantiate five, as a collection of five objects. As number systems elaborate, lengthy phrases like the fingers on one hand wear away to short words like five, which become symbolic through loss of the original phrasal form, likely supported in this truncation by the fingers' iconic/instantiative accompaniment (Overmann, 2021a). It's unparsimonious to think that WEIRD spoken numbers somehow bypassed a process of linguistic change still observable today in contemporary languages (Comrie, 2011). The likelihood of their having emerged similarly and shortened subsequently is supported by the relation of the words five and ten and terms for one/both hands in Proto-Indo-European (Beekes, 2011; Bomhard, 2008). That Proto-Indo-European spoken numbers were already well-truncated by 4500-2500 BCE highlights their greater antiquity, not an improbable symbolic emergence.

A similar transition was involved in realizing the numeral 5 from five linear marks. The numeral is symbolic_-its form and meaning are unrelated-but it is historically related to the marks, which were iconic/instantiative and thus non-symbolic, at least in the sense of arbitrariness. In the Babylonian and Egyptian writing systems, five linear marks were typically organized as three marks above two (Chrisomalis, 2010). Grouping marks in subitizable chunks gave their nonsubitizable total a greater visual appreciability. Over time, the total group of marks became recognized topologically as a function of the neurological reorganizations associated with literacy; topological recognition, in turn, let signs relax their forms to the point they were no longer recognizable, becoming symbolic (arbitrary) by losing their iconic/instantiative quality (Overmann, 2021b). Considered in light of the similar transition in spoken numbers, it seems 
reasonable to conclude, first, that much of the symbolling in WEIRD numbers has a non-symbolic basis, as symbolling generally does (Deacon, 2012), and second, that symbolling may not be either the process or the form whereby numerical meaning is initially conceived or expressed. By focusing on symbols, the embodied model excludes the antecedent non-symbolic representations from which they emerged, effectively kicking away the ladder used to get from the one to the other and leaving an inexplicable gap.

Fingers and notations are mentioned by the embodied model, but only as externalized representations of mentally created concepts. The concepts are themselves claimed to emerge from viewing and perhaps arithmetically manipulating collections of objects. The problem with this mechanism is twofold. First, it has the brain doing all the work of numerical conceptualization and elaboration, a flaw shared with the nativist and linguistic views. The human brain is a smart brain, comparatively speaking, but when it is positioned as the sole locus of numeracy, it receives more credit than it is perhaps due. Second, the mechanism misunderstands what a number is. The cardinality of a collection of objects is a property of that set, not a number itself, which is instead something that all sets of that cardinality have in common, the distinction between the threeness of a trio and three as a number that describes all trios (Russell, 1920). Here is a specific role for devices that makes them more than representations of externalized mental content: When a device like the fingers or a set of marks represents a quantity, that quantity becomes shared between the enumerated and reference sets of objects, affording opportunities to conceptualize cardinality shared between sets, a number (this is one of several roles for counting devices developed by the extended model).

The critical questions for the extended model are these: Where does "symbolling” start, and what does the historical development of numerical symbols from non-symbolic antecedents suggest about the role of symbolling in numeracy? And if material forms provide the incremental morphological change that enables numerical symbols to develop from non-symbolic antecedents, does it entail that material forms are something more than passive repositories of mental content? These are matters that suggest incorporating resources from the extended model in particular.

\section{The Extended Model}

In the extended model, numerical conceptualization starts with the perceptual ability to appreciate quantity and a world of appreciable quantity. The extended model diverges from the previous models both in viewing quantity perception as an integral system comprised of brain, body, and world and in seeing its material dimension as subject to alteration in ways that bring forth meaning. The critical difference between humans and other species is our use of materiality to make our innate numerical intuitions tangible, manipulable, and explicit (Coolidge \& Overmann, 2012; Malafouris, 2010). If using material forms is the mechanism by which number concepts become explicit, then as only humans use material forms for numerical purposes, only humans are likely to have numerical concepts in the way we understand them.

In contrast to the linguistic model, the extended model sees language as one of two interdependent, interacting, and distinct means of accessing and expressing numbers, the other being the manuovisual engagement of material forms. In facilitating access, use, and social transactability, language is viewed as a necessary component of numeracy but not the total mechanism whereby conceptual meaning emerges. Instead, the visual nature of numerosity and numeracy, in conjunction with the capacity of material forms to instantiate quantity and be 
manipulated into new configurations, yield a mechanism for making numerical intuitions visible and thus tractable to conceptualization, pattern recognition, and relational analyses. Expression by means of language is then a subsequent phenomenon that significantly enhances and expands the ability to acquire, use, and socially transact numerical concepts. Language and material forms are also viewed as interacting in ways that are both complementary and contradictory, either reinforcing one another to enhance a number system's stability over long spans of time or differing from the other with the potential to illuminate and explicate implicit properties and principles.

As was true of the embodied model, blending and symbolling remain important mechanisms for conceptualization and elaboration. However, the extended model conceives blends as adding the material domain as an input, with material forms anchoring and stabilizing the resultant concepts (Hutchins, 2005). The material component of the blend includes not just the objects enumerated, as the embodied model did, but also the devices and behaviors used for counting, which the embodied model omitted. Counting devices-distributed exemplars of appreciable quantity; the body; devices and behaviors that accumulate or accumulate and group; and handwritten notations - are critical inputs. They share cardinality with sets of enumerated objects in ways that potentialize the conceptualization of cardinalities shared between sets. Material properties like linearity, discreteness, manipulability, and concision also inform numerical properties. Properties can be explicitly provided by a material form used for counting, as when the fingers visually and conceptually define a quantity perceived as about three or four against the adjacent digits; properties can also be implicit, learned, or habituated, influencing the use of devices and the selection of new material forms through behaviors, beliefs, and expectations, as well as transferring structure between devices independent of their form.

The incorporation of new counting devices as an input to the blend provides opportunities for numerical concepts to acquire novel properties, functioning as the mechanism of elaboration. This mechanism contrasts with that of the embodied model, where elaboration was "a consequence of the systematic layering of metaphor upon metaphor, often over the course of centuries" (Lakoff \& Núñez, 2000, p. 47). In the extended model, new counting devices are recruited whenever the limitations of previous devices constrain numerical use: Fingers afford a small capacity for quantity and a finite persistence for representation. Devices that accumulate address these limitations by providing capacity and persistence, but impose new limitations, as their greater capacity can accumulate non-subitizable quantities to extents that are neither visually appreciable nor easily recounted. New limitations may eventually motivate the incorporation of yet other material forms, perhaps ones that not only accumulate but group to enhance visual appreciability and mitigate recounting. The result is a technological layering of material forms systematized by device affordances, and limitations that reliably and predictably emerge from the interaction of numerosity, material form, behaviors, and social needs.

The extended model not only adds material devices, it considers them an active component of the cognitive system for numbers. Their agency in this regard derives, in part, from their ability to influence our knowledge: They structure and organize numerical concepts in ways that inform both how we acquire them and what we understand them to be (Schlimm, 2018 points this out for notations; it is equally true of precursor technologies like fingers and tallies). Material forms also influence social behaviors: When we learn to use material forms for numbers, behaviors become socially patterned and individually habitual and automated. Patterned behaviors and associated expectations, in turn, can influence the use of a new material form in a habituated way, thereby transferring organization and structure between material forms. They influence us psychologically 
as well: In interacting with material forms, we develop familiarity and skill and acquire knowledge, all of which imply neurological change. Reading and writing involve specific neurological reorganizations (Dehaene et al., 2015), phenomena that include numerical notations. Changes in knowledge, behaviors, and psychological functioning then enable us to use and extend numbers, and to modify or add new material forms, especially over cultural spans of time. The task for each generation becomes simplified to a matter of learning to use the device (or set of devices) as a tool, in whatever form it was received.

The material forms used to represent and manipulate numbers have other elaborational effects, considered across device sequences. First, numerical information becomes increasingly concise. Representing the number ' 40 ' requires the fingers and toes of two people, 40 notches on a tally, four abacus beads worth ten each, or two Hindu-Arabic numerals. Concision of the explicit representational form is attended by an increase in the implicit knowledge that the user must supply (e.g., encoded value; positional weight). Concision affords numerical information a greater visual appreciability at greater volumes, making it more analytically tractable and increasing the recognition of patterns and relations. The net effect is this: The more devices there are in the elaborational sequence, the more numbers will differ from the perceptual experience of quantity in their content, structure, and organization.

Second, as new material forms are recruited to represent and manipulate numbers, concepts become distributed over the set of representational forms in a way that makes them irreducible to, and thus functionally independent of, any particular form. When distribution and independence are added to symbolling, the result is a perceived abstractness that obscures the conceptual system's material, non-symbolic roots. The ladder once used to transcend the perceptual experience of quantity and elaborate it as explicit concepts of symbolic number seemingly disappears, so we no longer realize we are still using one.

Finally, variability between cultural number systems, including anumeracy, becomes a function of whether or not a particular society uses material forms to represent and manipulate numerical information, which particular material forms it uses, and how it uses them. The need for numbers, and hence for using devices, is in turn modulated by social and demographic factors influencing internal complexity and/or external interactions, matters for which numbers are an adept and flexible management technology. Material culture may also have a more general effect. Independent of specific resourcing strategies, socio-material complexity might simply indicate a habitual materially based problem-solving and a socio-cultural environment wherein material forms distract from quotidian concerns, weaken cognitive biases, and provide unrelated/novel stimuli (German \& Barrett, 2005; Kirsh, 2014).

The critical questions for the extended model are these: Given its use of inference and the generally unobservable nature of historical change, must it also demonstrate empirical supportability, and if so, what form should this take? Is it critical to accept extension, or does the idea of a visual epistemology suffice? Do the isometries of form and function between written numerals and precursor technologies entail that if the former participates in extended states like reading, the latter should as well, and if so, how and why might the two differ in this regard? And when skeptics glibly reduce and dismiss extended cognition as panpsychism (Johnson \& Everett, 2021), how might the role of material forms as outlined above be effectively explained? 


\section{Conclusion}

As metaphor, Wittgenstein's ladder denotes a material structure whose form and function are determined by —and thus reflect - the needs, applications, and physiological, psychological, and behavioral capacities and capabilities of their users. As material structure, it also usefully seems to vanish at the appropriate point. The metaphor less usefully connotes a finished, manufactured artifact, since the material elaboration of numerosity as the conceptual domain of numbers is more accurately described as an incremental layering of material forms that solve predictable and recurrent limitations; influence the content, structure, and organization of the numerical concepts they represent and manipulate; demand users acquire the knowledge, skills, and neurological reorganizations needed to operate them; and assist them in that acquisition. The metaphor suggests the structure disappears, when in fact, it merely becomes invisible to us. The metaphor also suggests the starting and ending points differ, but may less aptly equate movement between the two with progress, when in actuality, getting from one to the other is the fairly predictable pathway that numerical elaboration tends to follow cross-culturally, as determined by the components and contexts interacting to produce it.

The material devices used to represent and manipulate numbers are not currently recognized by the nativist, linguistic, or embodied views as a component necessary to our elaboration of numerosity as numeracy. Instead, devices are assumed to be epiphenomenal, externalizations of mental content or assistants to internal mental activity. Yet their presence not only correlates reliably with numerical elaboration, their forms and use correlate reliably with differences in numerical content, structure, and organization. This suggests that the assumed causal directivity from mind to material — which leaves unexplained how the mind creates such concepts in the first place-be reversed to consider how the manuovisual engagement of material forms contributes to the historical realization of numerical concepts, just as it contributes to numerical conceptualization in contemporary mathematical practice. It also suggests that working across discipline boundaries has significant potential for helping to answer the critical questions that each perspective brings to the inquiry into numerical origins.

\section{References}

Amalric, M., \& Dehaene, S. (2016). Origins of the brain networks for advanced mathematics in expert mathematicians. Proceedings of the National Academy of Sciences of the United States of America, 113(18), 4909-4917.

Barth, H. C., Mont, K. La, Lipton, J., \& Spelke, E. S. (2005). Abstract number and arithmetic in preschool children. Proceedings of the National Academy of Sciences of the United States of America, 102(39), 14116-14121.

Beekes, R. S. P. (2011). The numerals. In Comparative Indo-European linguistics: An introduction (2nd ed., pp. 237-243). John Benjamins.

Bomhard, A. R. (2008). Some thoughts on the Proto-Indo-European cardinal numbers. In J. D. Bengtson (Ed.), In hot pursuit of language in prehistory. Essays in the four fields of anthropology in honor of Harold Crane Fleming (pp. 213-222). John Benjamins.

Brannon, E. M. (2005). The independence of language and mathematical reasoning. Proceedings of the National Academy of Sciences of the United States of America, 102(9), 3177-3178.

Cantlon, J. F., Brannon, E. M., Carter, E. J., \& Pelphrey, K. A. (2006). Functional imaging of numerical processing in adults and 4-y-old children. PLoS Biology, 4(5), 844-854. 
Chomsky, N. (1988). Language and problems of knowledge: The Managua lectures. MIT Press.

Chomsky, N. (2004). The generative enterprise revisited: Discussions with Riny Huybregts, Henk van Riemsdijk, Naoki Fukui and Mihoko Zushi (2nd ed.). Mouton de Gruyter.

Chrisomalis, S. (2010). Numerical notation: A comparative history. Cambridge University Press.

Comrie, B. (2011). Typology of numeral systems. Max Planck Institute. https://mpilingweb.shh.mpg.de/numeral/TypNumCuhk_11ho.doc

Coolidge, F. L., \& Overmann, K. A. (2012). Numerosity, abstraction, and the emergence of symbolic thinking. Current Anthropology, 53(2), 204-225. https://doi.org/10.1086/664818

Crollen, V., Mahe, R., Collignon, O., \& Seron, X. (2011). The role of vision in the development of fingernumber interactions: Finger-counting and finger-montring in blind children. Journal of Experimental Child Psychology, 109(4), 525-539.

Deacon, T. W. (2012). The symbol concept. In M. Tallerman \& K. R. Gibson (Eds.), The Oxford handbook of language evolution (pp. 393-405). Oxford University Press.

Dehaene, S. (2007). Symbols and quantities in parietal cortex: Elements of a mathematical theory of number representation and manipulation. In P. Haggard, Y. Rossetti, \& M. Kawato (Eds.), Sensorimotor foundations of higher cognition: Attention and performance (pp. 527-574). Oxford University Press.

Dehaene, S., Cohen, L., Morais, J., \& Kolinsky, R. (2015). Illiterate to literate: Behavioural and cerebral changes induced by reading acquisition. Nature Reviews: Neuroscience, 16(4), 234-244.

Dreyfus, T. (1991). Advanced mathematical thinking processes. In D. Tall (Ed.), Advanced mathematical thinking (pp. 25-41). Kluwer.

Evans, G. R. (1977). From abacus to algorism: Theory and practice in medieval arithmetic. British Journal for the History of Science, 10(2), 114-131.

Everett, D. L. (2005). Cultural constraints on grammar and cognition in Pirahã: Another look at the design features of human language. Current Anthropology, 46(4), 621-646.

Everett, D. L. (2007). Cultural constraints on grammar in Pirahã: A reply to Nevins, Pesetsky, and Rodrigues. [Unpublished Manuscript]. https://ling.auf.net/lingbuzz/000427

Everett, D. L. (2013). Recursion and human thought: Why the Pirahã don't have numbers. In J. Brockman (Ed.), Thinking: The new science of decision-making, problem-solving, and prediction (pp. 269-291). HarperCollins.

Fauconnier, G. (1997). Mappings in thought and language. Cambridge University Press.

Fauconnier, G., \& Turner, M. (2002). The way we think: Conceptual blending and the mind's hidden complexities. New York.

Fitch, W. T., Hauser, M. D., \& Chomsky, N. (2005). The evolution of the language faculty: Clarifications and implications. Cognition, 97(2), 179-210.

Frank, M. C., Everett, D. L., Fedorenko, E., \& Gibson, E. (2008). Number as a cognitive technology: Evidence from Pirahã language and cognition. Cognition, 108(3), 819-824.

Gallagher, S. (2013). The enactive hand. In Z. Radman (Ed.), The hand, an organ of the mind: What the manual tells the mental (pp. 209-225). MIT Press.

German, T. P., \& Barrett, H. C. (2005). Functional fixedness in a technologically sparse culture. Psychological Science, 16(1), 1-5.

Giaquinto, M. (2007). Visual thinking in mathematics: An epistemological study. Oxford University Press. 
Gilmore, C. K., McCarthy, S. E., \& Spelke, E. S. (2010). Non-symbolic arithmetic abilities and mathematics achievement in the first year of formal schooling. Cognition, 115(3), 394-406.

Gordon, P. (2004). Numerical cognition without words: Evidence from Amazonia. Science, 306(5695), 496-499.

Hauser, M. D., Chomsky, N., \& Fitch, W. T. (2002). The faculty of language: What is it, who has it, and how did it evolve? Science, 298(5598), 1569-1579.

Henrich, J., Heine, S. J., \& Norenzayan, A. (2010). The weirdest people in the world? Behavioral and Brain Sciences, 33(2-3), 61-135.

Howard, S. R., Avarguès-Weber, A., Garcia, J. E., Greentree, A. D., \& Dyer, A. G. (2018). Numerical ordering of zero in honey bees. Science, 360(6393), 1124-1126.

Hurford, J. R. (1987). Language and number: The emergence of a cognitive system. Blackwell.

Hurford, J. R. (2007). The origins of meaning: Language in the light of evolution. Oxford University Press.

Hutchins, E. (2005). Material anchors for conceptual blends. Journal of Pragmatics, 37(10), 1555-1577.

Johnson, M., \& Everett, C. (2021). Embodied and extended numerical cognition. In A. Killin \& S. AllenHermanson (Eds.), Explorations in archaeology and philosophy (pp. 125-148). Springer Nature.

Joseph, G. G. (2011). The crest of the peacock: Non-European roots of mathematics (3rd ed.). Princeton University Press.

Kaput, J. J. (1987). PME XI algebra papers: A representational framework. In E. Par, J. C. Bergeron, N. Herscovics, \& C. Kieran (Eds.), Proceedings of the 11th Psychology of Mathematics Education (PME) International Conference (Vol. 1, pp. 345-354). The International Group for the Psychology of Mathematics Education.

Karlsson, F. (2010). Syntactic recursion and iteration. Recursion and human language. In H. Van der Hulst (Ed.), Recursion and human language (pp. 43-67). Walter de Gruyter.

Kirsh, D. (2014). The importance of change and interactivity in creativity. Pragmatics \& Cognition, 22(1), 5-26.

Klein, J. (1992). Greek mathematical thought and the origin of algebra. Dover Publications, Inc.

Lakoff, G., \& Núñez, R. E. (2000). Where mathematics comes from: How the embodied mind brings mathematics into being. Basic Books.

Landy, D. (2010). Toward a physics of equations. In A. K. Goel, M. Jamnik, \& N. H. Narayanan (Eds.), Diagrammatic representation and inference: Proceedings of the 6th International Conference, Diagrams 2010, Portland, OR (pp. 160-166). Springer-Verlag.

Malafouris, L. (2010). Grasping the concept of number: How did the sapient mind move beyond approximation? In C. Renfrew \& I. Morley (Eds.), The archaeology of measurement: Comprehending heaven, earth and time in ancient societies (pp. 35-42). Cambridge University Press.

Malafouris, L. (2013). How things shape the mind: A theory of material engagement. MIT Press.

Malone, S. A., Pritchard, V. E., Heron-Delaney, M., Burgoyne, K., Lervåg, A., \& Hulme, C. (2019). The relationship between numerosity discrimination and arithmetic skill reflects the approximate number system and cannot be explained by inhibitory control. Journal of Experimental Child Psychology, 184, 220-231.

Nelsen, R. B. (1993). Proofs without words: Exercises in visual thinking (Vol. 1). The Mathematical Association of America.

Nevins, A., Pesetsky, D., \& Rodrigues, C. (2009). Evidence and argumentation: A reply to Everett (2009). 
Language, 85(3), 671-681.

Nieder, A. (2016). Representing something out of nothing: The dawning of zero. Trends in Cognitive Sciences, 20(11), 830-842.

Nieder, A. (2017a). Evolution of cognitive and neural solutions enabling numerosity judgements: Lessons from primates and corvids. Philosophical Transactions of the Royal Society of London. Series B, Biological Sciences, 373(1740), 20160514.

Nieder, A. (2017b). Number faculty is rooted in our biological heritage. Trends in Cognitive Sciences, 21(6), 403-404.

Núñez, R. E. (2011). No innate number line in the human brain. Journal of Cross-Cultural Psychology, 42(4), 651-668.

Núñez, R. E. (2017a). Is there really an evolved capacity for number? Trends in Cognitive Sciences, 21(6), 409-424.

Núñez, R. E. (2017b). Number-biological enculturation beyond natural selection. Trends in Cognitive Sciences, 21(6), 404-405.

Odifreddi, P. (1992). Classical recursion theory: The theory of functions and sets of natural numbers (J. Barwise, H. J. Keisler, P. Suppes, \& A. S. Troelstra (Eds.)). Elsevier.

Ott, D. (2009). The evolution of I-language: Lexicalization as the key evolutionary novelty. Biolinguistics, 3(2-3), 255-269.

Overmann, K. A. (2021a). Finger-counting and numerical structure. Frontiers in Psychology, 12, 723492. https://doi.org/10.3389/fpsyg.2021.723492

Overmann, K. A. (2021b). Writing system transmission and change: A neurofunctional perspective. In G. Gabriel, K. A. Overmann, \& A. Payne (Eds.), Signs - sounds - semantics. Nature and transformation of writing systems in the Ancient Near East (pp. 93-116). Ugarit-Verlag.

Parker, A. R. (2006). Evolving the narrow language faculty: Was recursion the pivotal step? In A. Cangelosi, A. D. M. Smith, \& K. Smith (Eds.), The evolution of language (pp. 239-246). World Scientific Publishing.

Piaget, J. (1928). Logique génétique et sociologie. Revue Philosophique de La France et de l'Étranger, 105, 167-205.

Pica, P., Lemer, C., Izard, V., \& Dehaene, S. (2004). Exact and approximate arithmetic in an Amazonian indigene group. Science, 306(5695), 499-503.

Pullan, J. M. (1968). The history of the abacus. Hutchinson \& Co.

Reuland, E. (2010). Imagination, planning, and working memory: The emergence of language. Current Anthropology, 51(S1), S99-S110.

Reynolds, B. E. (1993). The algorists vs. the abacists: An ancient controversy on the use of calculators. College Mathematics Journal, 24, 218-223.

Rotman, B. (1987). Signifying nothing: The semiotics of zero. Stanford University Press.

Roux, F., Boetto, S., Sacko, O., Chollet, F., \& Trémoulet, M. (2003). Writing, calculating, and finger recognition in the region of the angular gyrus: A cortical stimulation study of Gerstmann syndrome. Journal of Neurosurgery, 99(4), 716-727.

Russell, B. (1920). Introduction to mathematical philosophy (2nd ed.). George Allen \& Unwin, Ltd.

Sakel, J. (2012). Acquiring complexity: The Portuguese of some Pirahã men. Linguistic Discovery, 10(1), 
75-88.

Schlimm, D. (2018). Numbers through numerals: The constitutive role of external representations. In S. Bangu (Ed.), Naturalizing logico-mathematical knowledge: Approaches from psychology and cognitive science (pp. 195-217). Routledge.

Schulte, S. M. L. (2015). Writing the history of mathematical notation: 1483-1700. Docent Press.

Silver, D. S. (2017). The new language of mathematics. American Scientist, 105(6), 364-371.

Tang, Y., Zhang, W., Chen, K., Feng, S., Ji, Y., Shen, J., Reiman, E. M., \& Liu, Y. (2006). Arithmetic processing in the brain shaped by cultures. Proceedings of the National Academy of Sciences of the United States of America, 103(28), 10775-10780.

Taraban, R., \& Bandara, A. (2017). Beyond recursion: Critique of Hauser, Chomsky, and Fitch. Eastern European Journal of Psycholinguistics, 4(2), 58-66.

Varley, R. A., Klessinger, N. J. C., Romanowski, C. A. J., \& Siegal, M. (2005). Agrammatic but numerate. Proceedings of the National Academy of Sciences of the United States of America, 102(9), 3519-3524.

Wittgenstein, L. (1933). Tractatus Logico-Philosophicus. Kegan Paul, Trench, Trubner \& Co. 